\title{
Elaboração e validação de escala diagramática para quantificação da severidade de entomosporiose em folhas de pereira
}

\author{
Claudia Cardoso Nunes ${ }^{1}$, Silvio André Meirelles Alves ${ }^{2}$
}

\begin{abstract}
${ }^{1}$ Graduanda de Tecnologia em Fruticultura, Universidade Estadual do Rio Grande do Sul, Vacaria-RS, Bolsista FAPERGS. ${ }^{2}$ Embrapa Uva e Vinho, Rod. BR 285, km 115, CP 1543, CEP 95200-000, Vacaria-RS.

Autor para correspondência: Silvio André Meirelles Alves (silvio@cnpuv.embrapa.br)

Data de chegada: 06/10/2011. Aceito para publicação em: 01/08/2012.
\end{abstract}

\section{RESUMO}

Nunes, C.C.; Alves, S.A.M. Elaboração e validação de escala diagramática para quantificação da severidade de entomosporiose em folhas de pereira. Summa Phytopathologica, v.38, n.3, p.239-244, 2012.

A entomosporiose, causada pelo fungo Entomosporium mespili, é uma das principais doenças da pereira (Pyrus sp.) no Brasil. Considerandose a inexistência de métodos padronizados para quantificação dessa doença em folhas, o objetivo deste trabalho foi desenvolver uma escala diagramática para quantificação da severidade da entomosporiose da pereira. Para padronizar a avaliação, foi desenvolvida uma escala diagramática logarítmica com sete níveis $(0,3 ; 1,0 ; 2,3 ; 5,1 ; 11,1 ; 22,4$ e $40,0 \%$ de área foliar lesionada). Para a validação da escala, nove avaliadores estimaram a severidade de 60 imagens digitalizadas de folhas, com diferentes níveis de severidade. As avaliações foram feitas em duas etapas: sem e com o auxílio da escala. A severidade real foi obtida através do programa Quant v.1.0. A acurácia e a precisão dos avaliadores foram determinadas por regressão linear simples entre a severidade real e a estimada. A escala proposta proporcionou bons níveis de acurácia e precisão, boa repetibilidade das estimativas, além de rapidez e facilidade na avaliação, constituindo-se em uma ferramenta útil em estudos epidemiológicos e no desenvolvimento de estratégias de controle da entomosporiose da pereira.

Palavras-chave adicionais: Entomosporium mespili, doenças foliares, severidade, fitopatometria.

\begin{abstract}
Nunes, C.C.;Alves, S.A.M. Development and validation of a diagrammatic scale to quantify the severity of Fabraea leaf spot of pear. Summa Phytopathologica, v.38, n.3, p.239-244, 2012.

Fabraea leaf spot, caused by the fungus Entomosporium mespili, is one of the major diseases affecting pear (Pyrus sp.) in Brazil. Considering the lack of standardized methods to quantify this disease on leaves, the aim of this study was to develop a diagrammatic scale to quantify the severity of Fabraea leaf spot of pear. To standardize the assessment, we developed a diagrammatic logarithmic scale with seven levels $(0.3,1.0,2.3$, $5.1,11.1,22.4$ and $40.0 \%$ diseased leaf area). To validate the scale, nine raters estimated the severity of 60 scanned leaf images

at different severity levels. The evaluations were done in two steps: with and without the aid of the scale. The actual severity was obtained through the program Quant. v.1.0. The accuracy and the precision of raters were determined by simple linear regression between actual and estimated severity. The proposed scale provided good levels of accuracy and precision, good repeatability of estimates, and rapid and easy assessment, constituting thus a useful tool in epidemiological studies and for the development of strategies to control Fabraea leaf spot of pear.
\end{abstract}

Additional keywords: Entomosporium mespili, leaf diseases, severity, phytopathometry.

A entomosporiose, causada pelo fungo, Entomosporium mespili (DC.) Sacc. (forma perfeita: Fabraea maculata Atk.), é uma das principais doenças da pereira (Pyrus sp.) (9). Também conhecida como requeima, esta doença é caracterizada por apresentar pequenas lesões no limbo foliar e causar desfolha, o que afeta a capacidade fotossintética das plantas e reduz o rendimento.

Os sintomas são visíveis em ambas as faces das folhas jovens como lesões pequenas, avermelhadas a púrpuras, as quais coalescem, tornando-se marrom-escuras, podendo aparecer rodeadas por um halo clorótico; as folhas severamente infectadas apresentam aspecto necrótico, amarelecem e caem. Nos frutos há formação de manchas necróticas semelhantes às folhas, porém ficam deprimidas à medida que o fruto cresce, além disso, podem aparecer rachaduras, favorecendo a entrada de outros micro-organismos. A principal fonte de inóculo primário são as folhas caídas do ano anterior (6).

Essa doença ocorre na maioria das regiões produtoras do mundo (6) com maior severidade onde há verões quentes e úmidos (14). Por esse motivo, a importância da entomosporiose no Brasil é relativamente maior do que nos principais países produtores. O programa de melhoramento da pereira no Brasil busca desenvolver variedades adaptadas às condições locais e resistentes a entomosporiose (Oliveira, comunicação pessoal). ${ }^{1}$

Como a característica da entomosporiose é apresentar lesões de pequeno tamanho, a quantificação dessa doença se torna 
bastante dificultada e pouco precisa entre pessoas sem treinamento. Em função disso, uma escala diagramática para avaliar a severidade de entomosporiose constitui uma ferramenta necessária para aumentar a precisão das avaliações além de possibilitar compreender a doença sob diferentes fatores ambientais, suas curvas de progresso, os níveis de resistência do hospedeiro e a determinação de eficiência de produtos químicos.

As escalas diagramáticas constituem-se atualmente na principal ferramenta de avaliação da severidade de doenças (1, 10). Considerando-se a inexistência de métodos padronizados para quantificação de entomosporiose em folhas de pereira, o objetivo deste trabalho foi desenvolver uma escala diagramática para quantificação da severidade dessa doença e analisar os níveis de acurácia e precisão das estimativas geradas a partir de sua utilização.

Para elaboração da escala diagramática foram coletadas 200 folhas de pereira das cultivares Abate Fetel, Packham's Triumph, William's e Rocha em pomares experimentais localizados nos municípios de Vacaria-RS, Fraiburgo-SC e São Joaquim-SC. Foram coletadas folhas com diferentes níveis de severidade durante os meses de outubro de 2010 a maio de 2011. As folhas foram fotografadas com câmera digital e, através do programa Quant v.1.0 (12), determinou-se a área foliar sadia e a área lesionada. Os limites da escala foram definidos como a mínima e a máxima proporção da área foliar lesionada. Os níveis intermediários foram estabelecidos em função da forma e distribuição mais frequentes das lesões, segundo incrementos logarítmicos, respeitando as limitações da acuidade visual humana com base na lei de Weber-Fechner (11).

Para o teste de acuidade visual e validação da escala foram selecionadas 60 imagens digitais de folhas com entomosporiose com diferentes níveis de severidade. A severidade foi estimada por nove avaliadores, sendo cinco sem experiência e quatro com experiência, em duas etapas: inicialmente foi realizada uma avaliação sem uso da escala; em seguida, as mesmas imagens foram apresentadas em uma nova sequência, e realizou-se a avaliação com o auxílio da escala.

A acurácia e a precisão dos avaliadores foram determinadas por regressão linear simples (10) entre a severidade real, obtida eletronicamente, como variável independente e a severidade estimada pelo avaliador, como variável dependente.

A acurácia das estimativas foi determinada pelo teste $t$ aos níveis de 1 e $5 \%$ de probabilidade, aplicada ao intercepto da regressão linear $(a)$ para verificar se este foi significativamente diferente de 0 e ao coeficiente angular da reta $(b)$ para testar se foi significativamente diferente de 1 . Valores de intercepto significativamente diferentes de zero indicam, em níveis baixos de intensidade da doença, superestimativa $(>0)$ ou subestimativa $(<0)$ da severidade real, enquanto valores de coeficiente angular da reta que diferem de 1 indicam superestimativa $(>1)$ ou subestimativa $(<1)$ sistemática da severidade real em todos os níveis de intensidade da doença (11). A precisão das estimativas foi obtida pelo coeficiente de determinação da regressão $\left(R^{2}\right)$ e pela variância dos erros absolutos (severidade estimada severidade real).

O nível máximo de severidade observado nas amostras foi de $40 \%$. Nas pereiras europeias utilizadas para as coletas das folhas, raramente foram encontradas severidades maiores do que essa, em condições de campo. A escala diagramática para entomosporiose da pereira foi elaborada com sete níveis de severidade, representados pelos valores de 0,$3 ; 1,0 ; 2,3 ; 5,1$; 11,$1 ; 22,4$ e $40,0 \%$ de área foliar lesionada considerando-se os limites mínimo e máximo de severidade (Figura 1).

Nos níveis de severidade acima de $11,1 \%$ a representação dos sintomas incluiu lesões coalescidas, como adotado na elaboração da escala diagramática para cercosporiose da alface (4).

As análises para validação mostraram que dentre os nove avaliadores, sem o auxílio da escala, oito foram pouco acurados, pois apresentaram valores de intercepto $(a)$ significativamente diferente de zero, com valores médios de todos os avaliadores de 4,76 (Tabela 1, Figura 2). Com a utilização da escala, todos os nove avaliadores melhoraram os níveis de acurácia, com o valor médio de intercepto de $-0,33$, não diferindo estatisticamente de zero.

Sem o auxílio da escala o coeficiente angular da reta $(b)$ variou de 1,03 a 1,89 e a média dos avaliadores foi de 1,39, significativamente diferente de $1(\mathrm{p}<0,01 ; \mathrm{p}<0,05)$. Ao passo que, com a utilização da escala, o coeficiente angular variou de 0,96 a 1,17, e a média entre os avaliadores foi de 1,06 .

Dentre os avaliadores, apenas o avaliador $H$ não superestimou a severidade, indicando a presença de desvios positivos constantes para a maioria dos avaliadores (Figura 3). A tendência dos avaliadores em superestimar os níveis de severidade é frequente em estudos envolvendo validação de

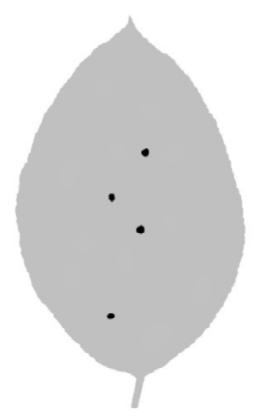

$0,3 \%$

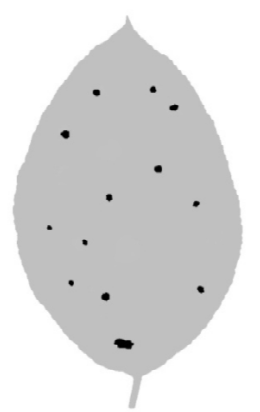

$1,0 \%$

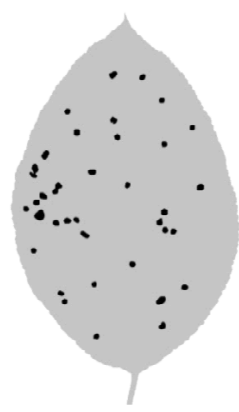

$2,3 \%$

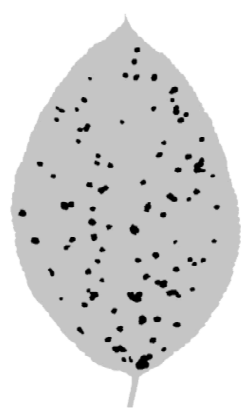

$5,1 \%$

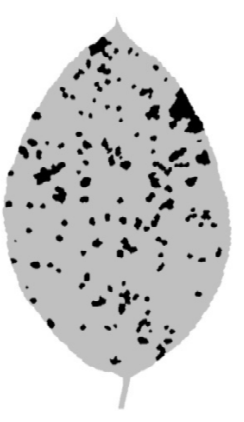

$11,1 \%$

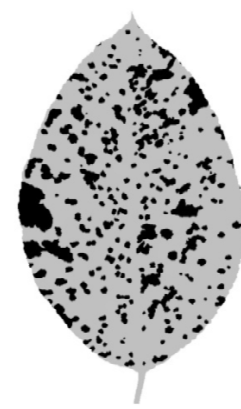

$22,4 \%$

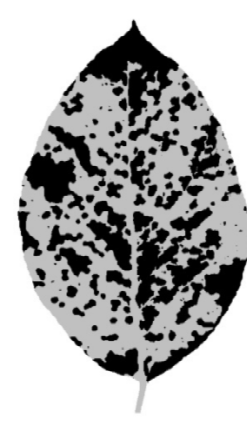

$40,0 \%$

Figura 1. Escala diagramática com sete níveis para quantificação da severidade de entomosporiose (Entomosporium mespili) em folhas de pereira (Pyrus sp.). 
Tabela 1. Intercepto $(a)$, coeficiente angular da reta $(b)$ e coeficiente de determinação $\left(R^{2}\right)$ de equação de regressão linear simples relacionando estimativas visuais de entomosporiose em folhas de pereira efetuadas por nove avaliadores (sem e com auxílio da escala diagramática) à severidade real determinada eletronicamente.

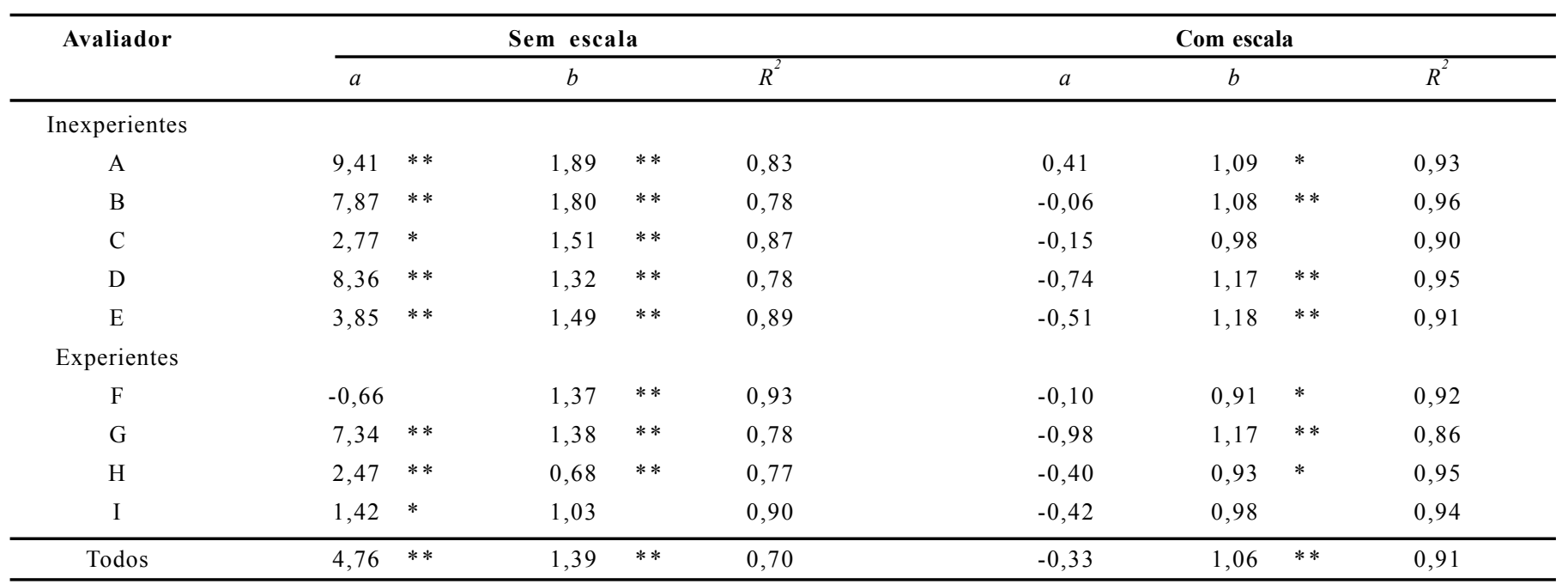

*Asterisco indica que a hipótese de nulidade $(\mathrm{a}=0$ ou $\mathrm{b}=1)$ foi rejeitada pelo teste $\mathrm{t}(\mathrm{p}<0,05)$. ** Asterisco indica que a hipótese de nulidade $(\mathrm{a}=0$ e $\mathrm{b}=1)$ foi rejeitada pelo teste $\mathrm{t}(\mathrm{p}<0,01)$
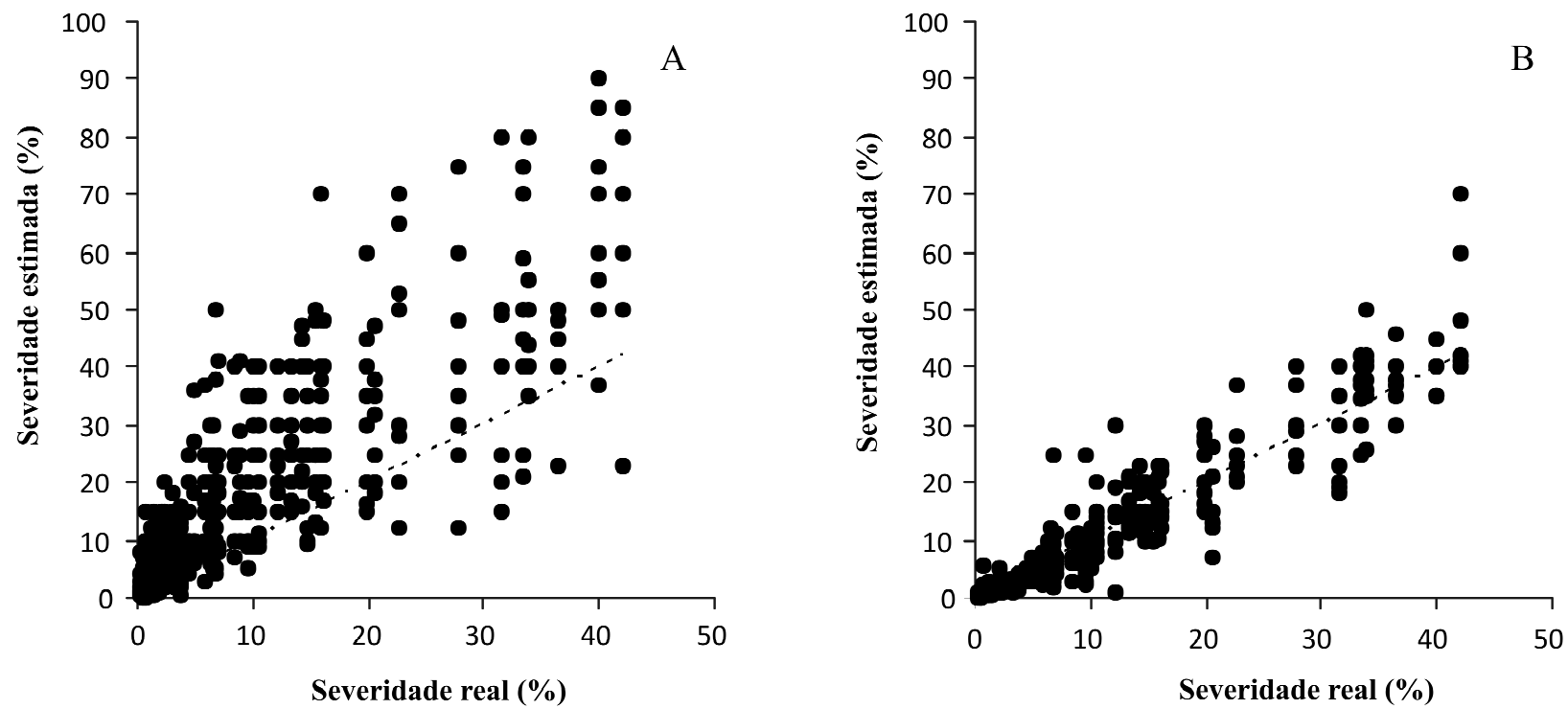

Figura 2. Severidade estimada por nove avaliadores sem o auxílio (A) e com auxílio da escala diagramática (B) para avaliação de entomosporiose em folhas de pereira. A linha cheia representa a regressão linear entre a severidade real e a estimada. A linha tracejada representa o ajuste ideal em que a severidade real é igual à estimada.

escalas diagramáticas $(2,3,7,8)$.

Existe uma tendência natural das pessoas a superestimarem as doenças porque elas estão avaliando a doença e não o tecido sadio. Além disso, há uma ilusão proporcionada pela relação entre o tamanho e número das lesões, dessa forma folhas com muitas lesões de pequeno tamanho parecem ter mais doença que aquelas com poucas lesões de tamanho maior (13).

O coeficiente de determinação da regressão pode ser utilizado para avaliar a precisão, definida como a exatidão de uma operação onde há rigor ou refinamento na medida (1). Sem a utilização da escala diagramática, o coeficiente de determinação variou de 0,77 a 0,93 . A precisão média obtida pela regressão conjunta de todos os avaliadores foi relativamente baixa, com 0,70 (Tabela 1), enquanto que com o auxílio da escala, o coeficiente de determinação variou de 0,86 a 0,96 .

Oito avaliadores melhoraram a precisão das estimativas, uma vez que maiores valores de $R^{2}$ foram obtidos com o uso da escala, com média de 0,91 (Figura 4). Outros estudos também indicam melhoria na precisão com a utilização de escalas diagramáticas demonstrando sua importância em estudos de quantificação de doenças $(2,3,4,5,7,8)$.

Sem o auxílio da escala, a variação dos erros absolutos foi de $-39,92$ a 43,29, e com a escala, a variação foi de $-14,99$ a 26,03. De acordo com Nutter Jr. (11), para que um avaliador seja considerado excelente, o erro de suas estimativas deve estar em um intervalo de $\pm 5 \%$ do valor real, e não pode ultrapassar $\pm 10 \%$ para ser considerado bom. No presente trabalho, num total de 540 estimativas geradas, na avaliação realizada sem o 

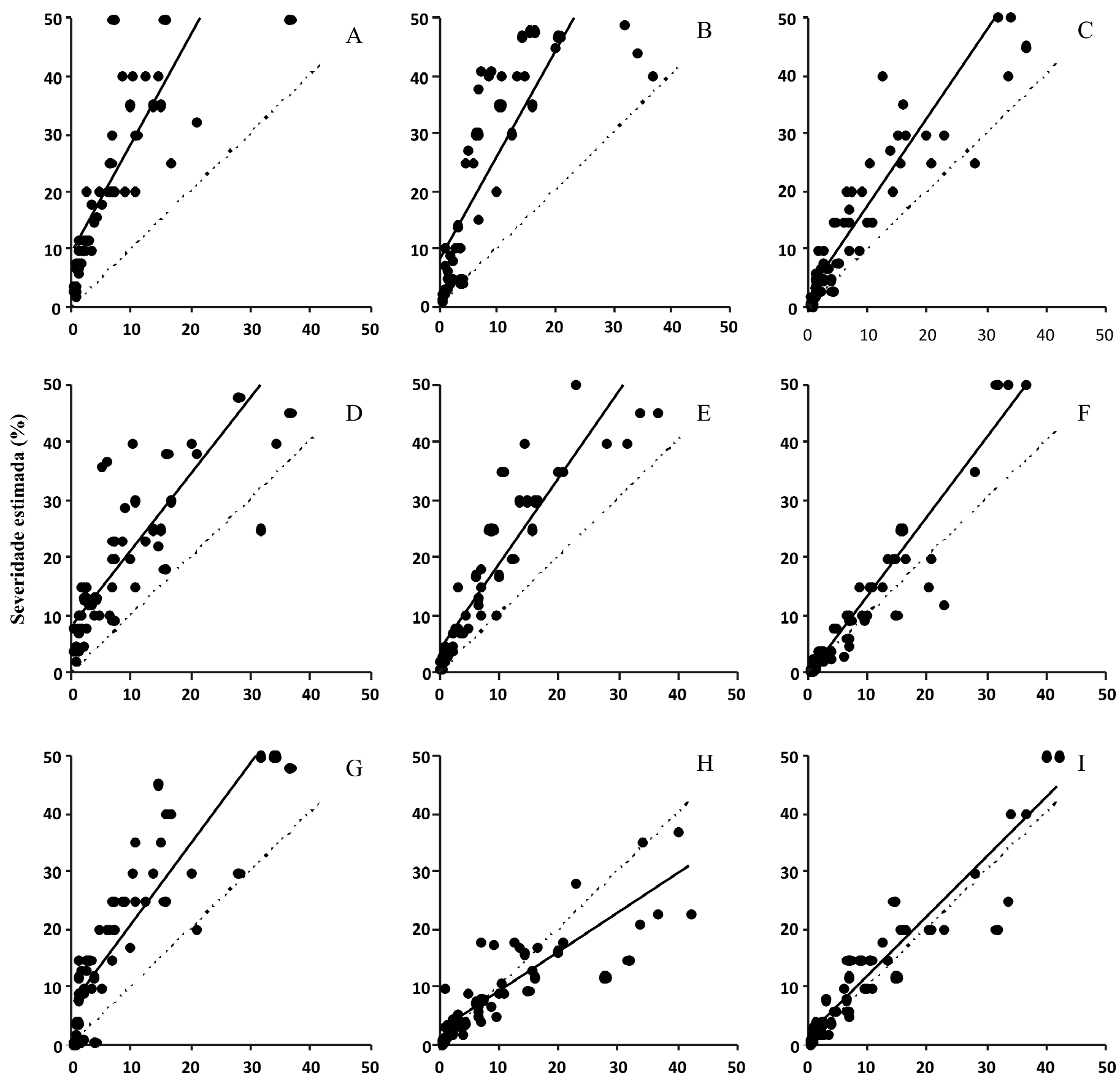

Severidade real $(\%)$

Figura 3. Severidade estimada por nove avaliadores sem o auxílio da escala diagramática para entomosporiose em folhas de pereira. A linha cheia representa a regressão linear entre a severidade real e a estimada. A linha tracejada representa o ajuste ideal em que a severidade real é igual à estimada.

auxílio da escala diagramática, verificou-se que 102 apresentaram erros superiores a $10 \%$, no entanto, com o auxílio da mesma, apenas 14 estimativas apresentaram erros superiores a esta faixa (Figura 5). Os desvios foram mais evidentes para níveis de severidade acima de 5\%, não havendo um padrão definido indesejável.

A utilização da escala diagramática proporcionou bons níveis de acurácia e precisão das estimativas, além de rapidez e facilidade na avaliação, constituindo assim uma ferramenta útil em estudos epidemiológicos do patossistema E. mespili - pereira e de desenvolvimento de estratégias de controle da doença.

\section{AGRADECIMENTOS}

Os autores manifestam agradecimento a todos os avaliadores 

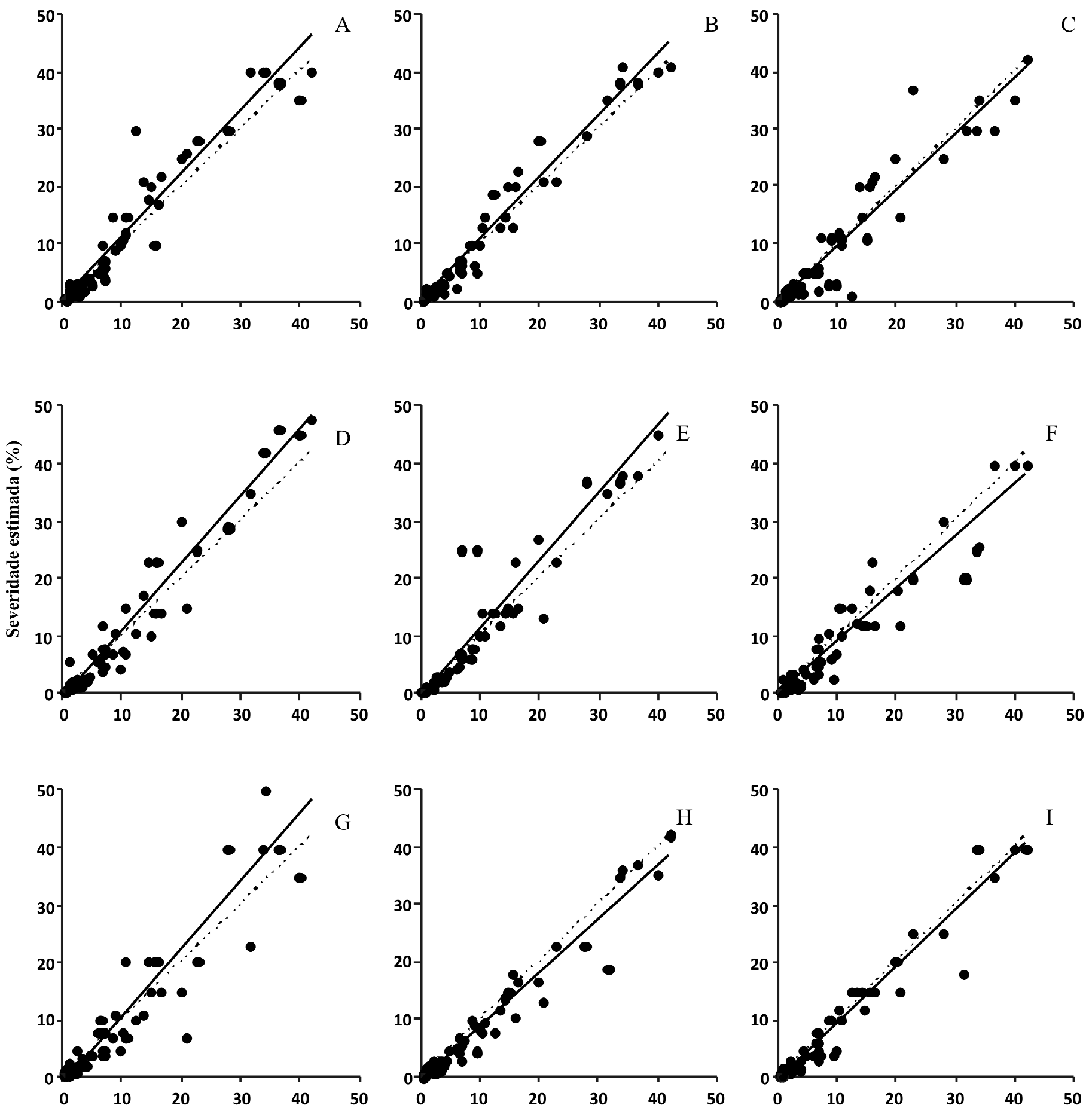

Severidade real $(\%)$

Figura 4. Severidade estimada por nove avaliadores com o auxílio da escala diagramática para entomosporiose em folhas de pereira. A linha cheia representa a regressão linear entre a severidade real e a estimada. A linha tracejada representa o ajuste ideal em que a severidade real é igual à estimada.

que participaram das etapas de validação da escala, bem como à Fundação de Amparo à Pesquisa do Estado do Rio Grande do Sul - FAPERGS, à Embrapa Uva e Vinho, Estação Experimental de Fruticultura de Clima Temperado, Vacaria-RS, à Empresa de Pesquisa Agropecuária e Extensão Rural de Santa Catarina EPAGRI, Estação Experimental de Fruticultura de São Joaquim$\mathrm{SC}$ e à Empresa Fischer $\mathrm{S} / \mathrm{A}$, Fraiburgo-SC.

\section{REFERENCIAS BIBLIOGRÁFICAS}

1. Amorim L. Avaliação de doenças. In: Bergamin Filho A, Kimati H, Amorim L. Manual de fitopatologia: princípios e conceitos. 3. ed. São Paulo: Ceres, 1995. v.1, cap. 32, p. $647-$ 671

2. Celoto, M.I.B.; Papa, M.F.S. Elaboração e validação de escala diagramática para quantificação da mancha alvo em folhas de 

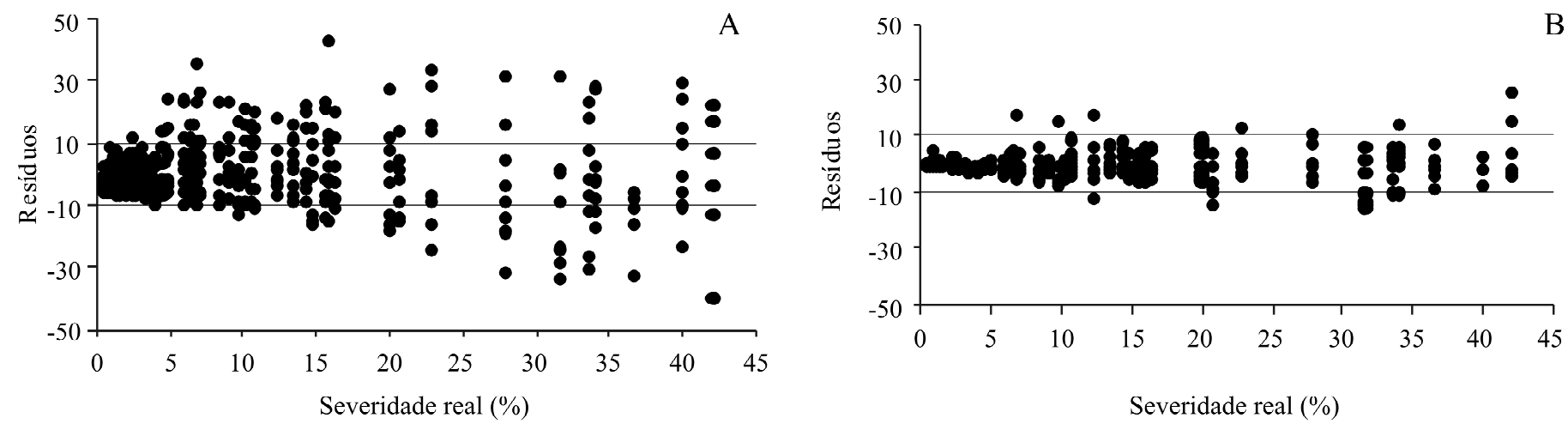

Figura 5. Resíduos (severidade estimada - severidade real) das estimativas de entomosporiose em folhas de pereira sem o auxílio (A) e com auxílio da escala diagramática (B). As duas linhas paralelas indicam a faixa de erros das estimativas no intervalo de $\pm 10 \%$.

acerola. Tropical Plant Pathology, Lavras, v.35, n.4, p.258$262,2010$.

3. Fischer, I.H.; Alves, S.A.M.; Almeida, A.M.; Arruda, M.C.; Bertani, R.M.A.; Garcia, M.J.M. Elaboração e validação de escala diagramática para quantificação da severidade da antracnose em frutos de maracujá amarelo. Summa Phytopathologica, Jaboticabal, v.35, n.3, p.226-288, 2009.

4. Gomes, A.M.A.; Michereff, S.F.; Mariano, R.L.R. Elaboração e validação de escala diagramática para cercosporiose da alface. Summa Phytopathologica, Botucatu, v. 30 , n.1, p.38$42,2004$.

5. Hirano, M.; Hikishima; Silva, A.J.; Xavier, S.A.; Giovanetti, C. Validação de escala diagramática para estimativa de desfolha provocada pela ferrugem asiática em soja. Summa Phytopathologica, Botucatu, v.36, n.3, p.248-250, 2010.

6. Jones, A.L.; Aldwinckle, H.S. Compendium of apple and pear diseases. St. Paul: The American Phytopathological Society, 1990. 100p.

7. Leite, R.M.V.B.C.; Amorim, L. Elaboração e validação de escala diagramática para mancha de Alternaria em girassol. Summa Phytopatologica, Botucatu, v. 28, n.1, p.14-19, 2002.

8. Michereff, S.J.; Noronha, M.A.; Andrade, D.E.G.T.; Oliveira, E.P.; Xavier Filha, M.S.; Moreira, P.A.A. Development and validation of a diagrammatic key for Cercospora leaf spot of sweet pepper. Summa Phytopathologica, Botucatu, v.32, n.3, p.260-266, 2006 .

9. Nakasu, B.H.; Herter, F.G.; Camelatto, D.; Reisser Júnior, C.;
Fortes, J.F.; Castro, L.A.S.; Raseira, A.; Freire, C.J.S.; Faoro, I.; Petri, J.L.; Leite, G.B.; Pereira, J.F.M.; Cantillano, R.F.F.; Veríssimo, V.; Simões, F. A cultura da pera. Brasília: Embrapa Clima Temperado; Embrapa Informação Tecnológica, 2007. 58 p. (Coleção Plantar, 58).

10. Nutter Jr., F.W.; Esker, P.D.; Coelho Netto, R.A. Disease assessment concepts and the advancements made in improving the accuracy and precision of plant disease data. European Journal of Plant Pathology, Dordrecht, v.115, n.1, p.95-103, 2006 .

11. Nutter Jr, F.W.; Schultz, P.M. Improving the accuracy and precision of disease assessment: selection of methods and use of computer-aided training programs. Canadian Journal of Plant Pathology, Ottawa, v.17, n.2, p.174-184, 1995.

12. Vale, F.X.R.; Fernandes Filho, E.I.; Liberato, J.R. A software plant disease severity assessment. In: International Congress of Plant Pathology, 8., 2003, Christchurch. Anais. Christchurch: New Zealand, 2003.p.105 (Resumo).

13. Vale, F.X.R.; Jesus Junior, W.C.; Liberato, J.R.; Souza, C.A. Quantificação de doenças e do crescimento do hospedeiro. In: Vale, F.X.R.; Jesus Junior, W.C.; Zambolin, L. Epidemiologia aplicada ao manejo de doenças de plantas. Belo Horizonte: Perffil, 2004. cap 3, p. 91-121.

14. Zwet, T. van der. Effects of cultural conditions on sporulation, germination, and pathogenicity of Entomosporium maculatum. Phytopathology, St. Paul, v.75, n.1, p.94-97, 1985. 